\section{Erfolg der Telemedizin steht und fällt mit dem Breitbandausbau}

Wenn es um die Digitalisierung geht, ist Deutschland Schlusslicht in Europa. Dass es im Gesundheitswesen digital nicht so recht voran geht, liegt nicht nur an Datenschutzbedenken und an Widerständen aus

der Ärzteschaft, sondern auch am stockenden Breitbandausbau.
Die Diabetologie ist ein datengetriebenes Fach, auf welches das E-Health-Gesetz unmittelbar Einfluss haben wird. So könnte der elektronische Medikationsplan die medikamentöse Einstellung multimorbider Typ-2-Diabetiker erleichtern, während das „Patientenfach“ auf der elektronischen Gesundheitskarte sich zum Sammeln von Daten aus Wearables und Glukosemesssystemen nutzen lasse. „So langsam bewegt sich auf diesem Gebiet auch etwas“, sagte Manuel Ickrath, Berater aus Wiesbaden und Sprecher der Anfang 2017 gegründeten Task Force Digitalisierung in der Deutschen Diabetes-Gesellschaft (DDG).

Doch der Teufel steckt wie so oft im Detail: „Es ist nicht klar, in welchen Formaten die Daten aus Wearables eingespeichert werden“, mahnte Ickrath, „geschweige denn wie viele Daten das eigentlich sind." Probleme gebe es auch bei der Interoperabilität: „Die mangelnde Konnektivität der verschiedenen Devices ist aktuell die Achillesferse der DDG und der Hauptgrund, warum immer noch viele Diabetologen der Digitalisierung zögerlich gegenüberstehen." Gemeint sind Bilder vom Kabelsalat an einem Praxistresen, wie sie wohl jeder schon bei Kongressen auf Vortragsfolien gesehen hat. Hier können die Patienten über unzählige verschiedene Ports ihre diversen Blutzuckmessgeräte, CGM-Systeme oder Insulinpumpen zur Datenübertragung andocken.

\section{Die Politik muss ihre Hausaufgaben machen}

Auch eine gemeinsame Plattform zur herstellerunabhängigen Datenauswertung lässt bislang auf sich warten. „Ganz aktuell hat ein Anbieter sine Software für alle Systeme geöffnet - das hat hoffentlich Sogwirkung“, erklärte Ickrath. Im nächsten Schritt will die Task Force Gespräche mit den verschiedenen PVS-Anbietern führen, damit in Zukunft auch die Anbindung der Diabetessoftware an die Praxissoftware gelingt. Ickrath machte klar, dass für den Erfolg der Digitalisierung im Gesundheitswesen aber auch die Politik ihre Hausaufgaben machen muss: „Wie sollen die Vorteile der Digitalisierung und der Telemedizin genutzt werden, wenn in Deutschland der Breitbandausbau stockt und Deutschland in diesem Punkt Schlusslicht in Europa ist?"

Umso intensiver engagiert sich derzeit die DDG in der Politik - und zwar nicht nur auf dem Gebiet der Digitalisierung, sondern auch in der sektorenübergreifenden Qualitätssicherung, wie sie das Institut für Qualität und Transparenz im Ge- sundheitswesen (IQTiG) gewährleisten soll. Wie Prof. Baptist Gallwitz berichtete, der als ehemaliger DDG-Präsident innerhalb der DDG hauptverantwortlich für Stellungnahmen der DDG an das IQTiG ist, kritisiert die Fachgesellschaft zum einen, dass für die Arbeit des neuen Instituts bislang keine strukturierte und prozedural festgelegte Einbindung der wissenschaftlichen Fachgesellschaften oder anderer patientennaher Berufsgruppen vorgesehen ist. Deshalb kann die DDG nur aus der Ferne verfolgen, welche Qualitätsindikatoren im IQTiG derzeit erarbeitet werden und anhand derer die Qualität von Einrichtungen beurteilt werden soll. „Die Indikatoren, die bislang bekannt sind, berücksichtigen eher Randbereiche, die nicht flächendeckend messbar sind“, kritisierte Prof. Gallwitz. Demgegenüber fehlten wichtige und leicht messbare Indikatoren wie die Zahl der Majoramputationen.

DDG: Wir werden gehört, nun geht's an die Umsetzung Auch was die Weiterentwicklung des Arzneimittelmarktneuordnungsgesetz (AMNOG) angeht, mischt die DDG kräftig mit. Der neue DDG-Präsident Prof. Dirk Müller-Wieland berichtete: „Wir haben in den vergangenen Jahren erreicht, dass wir gehört werden - jetzt geht es an die Umsetzung, und die ist ungleich komplizierter." Das 2011 in Kraft getretene AMNOG regelt die Preisbindung für zugelassene Arzneimittel, und zwar nach den Vorgaben des Instituts für Qualität und Wirtschaftlichkeit im Gesundheitswesen (IQWiG). „Wenn diese Regeln nicht dem wissenschaftlichen Stand entsprechen, muss eben nachgebessert werden“, forderte Prof. Müller-Wieland.

Problematisch sei allerdings, dass nicht eindeutig definiert ist, wer den medizinischen Standard festlegt, an dem sich Vergleichstherapien messen lassen müssen. „Auch die Festlegung der Fragestellung im Rahmen einer Nutzenbewertung ist extrem wichtig“, erklärte Prof. Müller-Wieland, „denn wenn man gefragt wird, ob eine Wand schwarz oder weiß ist, hat man nun einmal keine Chance, ,orange' zu antworten!“ Einzig die Prozedur einer Nutzenbewertung sei festgelegt, und damit gelte sie als gesetzeskonform. „Es fehlt aber die Plausibilitätskontrolle so etwas würden wir in keinem anderen Lebensbereich akzeptieren“, mahnte der DDG-Präsident.

Quelle: Sitzung „Neue Gesetze: Bedeutung für die Diabetesversorgung“, Jahrestagung der DDG am 27. 5. 2017 in Hamburg 